Musées, Patrimoine et Culture scientifiques et techniques

$173 \mid 2017$

septembre-octobre 2017

\title{
Évaluation par les publics et les médiateurs de l'exposition du muséum de Toulouse Les \\ Savanturiers
}

Alric Amirault et Thomas Rokita

\section{OpenEdition}

Journals

Édition électronique

URL : http://journals.openedition.org/ocim/1848

DOI : $10.4000 /$ ocim. 1848

ISSN : 2108-646X

Éditeur

OCIM

Édition imprimée

Date de publication : 1 septembre 2017

Pagination : 5-11

ISSN : 0994-1908

\section{Référence électronique}

Alric Amirault et Thomas Rokita, «Évaluation par les publics et les médiateurs de l'exposition du muséum de Toulouse Les Savanturiers », La Lettre de I'OCIM [En ligne], 173 | 2017, mis en ligne le 01 septembre 2018, consulté le 01 mai 2019. URL : http://journals.openedition.org/ocim/1848 ; DOI : 10.4000/ocim. 1848

Ce document a été généré automatiquement le 1 mai 2019.

Tous droits réservés 
Évaluation par les publics et les médiateurs de l'exposition du muséum de Toulouse Les

\section{Savanturiers}

Alric Amirault et Thomas Rokita

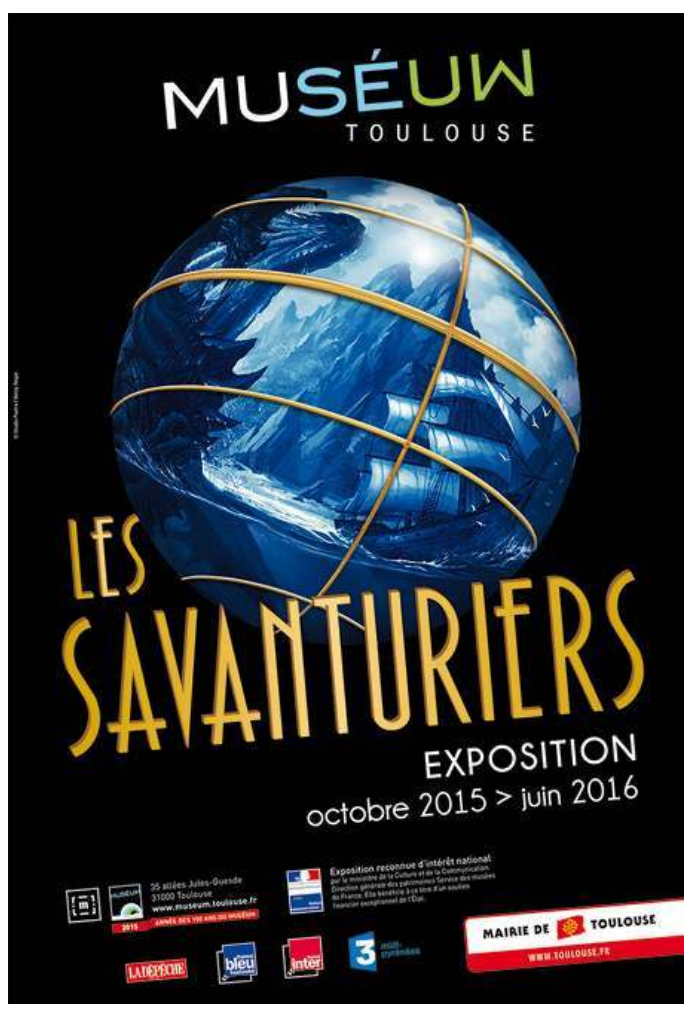

(c) Muséum de Toulouse/Studio Pastre/Rémy Régat 
1 Pionnier dans les découvertes scientifiques sur l'origine préhistorique de l'Homme, ce qui lui a valu une reconnaissance internationale, le Muséum de Toulouse se définit depuis 1865 comme un carrefour entre le monde des sciences, celui de la culture, les enjeux et les questions de société. Il articule ses missions avec différents espaces proposant une exposition permanente, une exposition temporaire et une exposition dite "dossier" depuis sa réhabilitation en 2008. Conçu comme un outil de partage et de critique des savoirs - lors de l'exposition Ours, mythes et réalités en 2014, le visiteur était amené à se forger sa propre opinion sur la cohabitation inter-espèces - c'est une plateforme d'information et de débats sur la relation Homme-Nature-Environnement, qui correspond à la muséologie de point de vue telle que définie par Jean Davallon ${ }^{1}$.

2 L'exposition analysée dans cet article s'intitule Les Savanturiers, et s'est tenue d'octobre 2015 jusqu'en août 2016. Elle avait pour objectif de célébrer les 150 ans du muséum de Toulouse en mettant à l'honneur les figures, célèbres ou anonymes, qui en ont composé le patrimoine scientifique. Par extension, elle amenait à penser l'avenir des collections muséales en créant un pont entre passé et présent. Invitant le grand public à voyager à travers ses collections, le muséum abolissait ainsi les frontières disciplinaires et mêlait sensibilité et réflexion au cœur d'une scénographie labyrinthique.

En passant d'une salle à une autre, avec un thème propre à chacune, le visiteur parcourait l'espace et le temps via des dispositifs variés : un périscope pour visualiser des photographies dans "La Cabine", des volants à tourner pour faire apparaître des textes et images, mais surtout "Le Vaisseau" qui fournissait une reconstitution 3D à partir d'ossements, d'un lion des cavernes disparu il y a 18500 ans. Ce dernier dispositif a d'ailleurs été récompensé du prix "Patrimoine et innovations" dans la catégorie "dispositifs d'immersion", lors des $7^{\text {es }}$ Rencontres nationales Culture et Innovation de $2016^{2}$.

\section{Méthodologie globale}

4 Les statistiques permettent depuis de nombreuses années de nommer diverses tendances à partir de chiffres de fréquentation, de mesurer l'impact des politiques culturelles, ou encore la satisfaction des visiteurs ${ }^{3}$. Ce que vient compléter le traitement qualitatif de la réception des publics en développant une réflexion à propos de la médiation entre l'objet culturel et le visiteur.

5 C'est dans cette dernière optique que se positionne la présente étude ${ }^{4}$. Lors de sa préparation plusieurs axes ont rapidement émergé : la fictionnalisation - comprendre la mobilisation de la fiction - et la communication ; les horizons d'attente et la réception de l'exposition ; le parcours de visite et les usages des dispositifs ; la médiation. En vue de traiter les problématiques précitées, nous avons eu recours à des méthodologies qualitatives plurielles : analyses sémiopragmatiques pluri-média, observations participantes ou non, entretiens semi-directifs, entretiens en re-situ subjectif. Cette étude s'est voulue qualitative, tant par le choix d'un nombre restreint d'informations recueillies, que par la précision du traitement de celles-ci.

\section{Communication et fictionnalisation}

6 Pour mener notre réflexion autour de la communication et la fictionnalisation de l'exposition, nous avons réalisé une analyse sémiopragmatique des supports de 
communication suivants : affiche, parcours de visite, dépliant. Nous avons analysé la communication web du muséum à propos des Savanturiers sur le site officiel du muséum, ainsi que ses comptes Youtube, Facebook et Twitter. Aussi, nous avons analysé un corpus d'articles de presse numérique en tapant les mots clés "les savanturiers toulouse" dans le moteur de recherche Google, en janvier 2016, ne gardant que la première page de résultats. Enfin, nous avons confronté nos analyses aux discours du concepteur et scénographe de l'exposition Stéphane Mountels, et de Marie-Pierre Pastre, directrice du Studio Pastre, chargée de la communication autour de l'exposition.

Le plan de visite de l'exposition Les Savanturiers

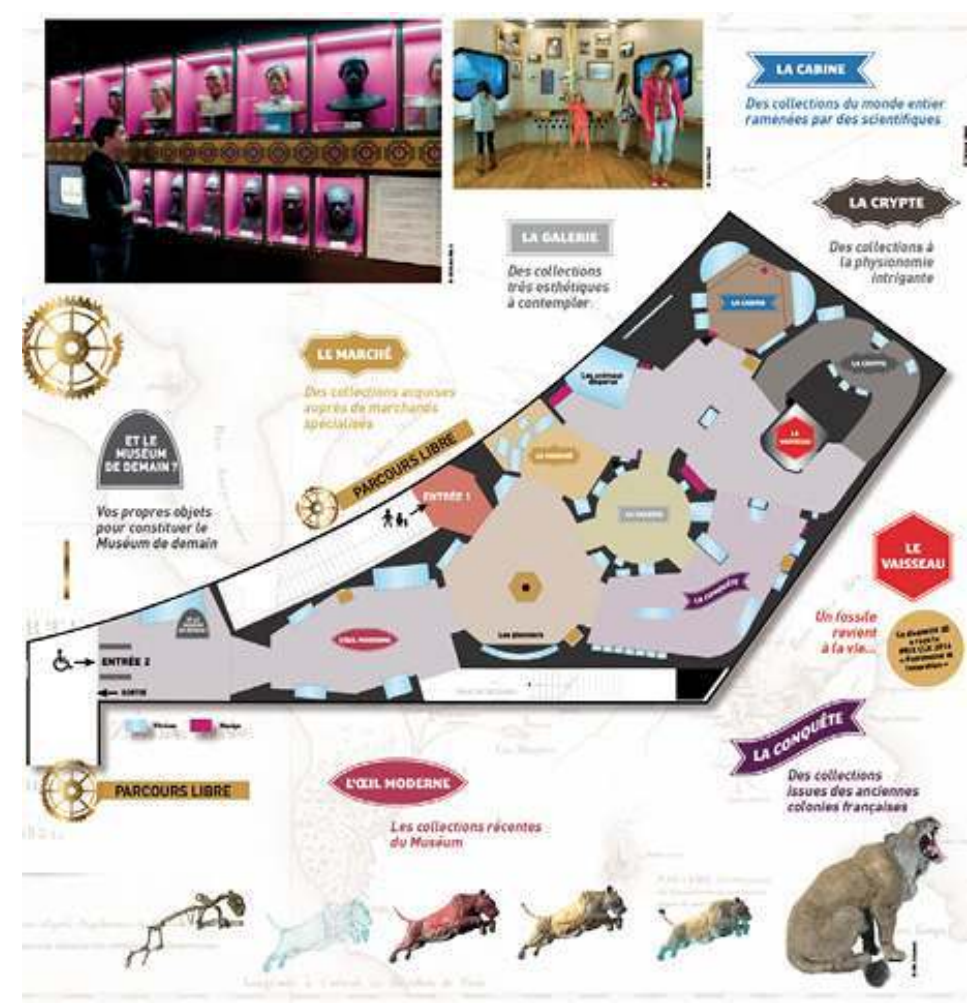

(c) Muséum de Toulouse/Studio Pastre

7 La lecture des différents supports de communication nous a tout d'abord questionnés sur les intentions de l'exposition Les Savanturiers. Comment la fictionnalisation de ses collections a-t-elle notamment été mobilisée dans la communication de l'exposition ? Le muséum s'est adapté aux évolutions muséographiques en résonance avec notre époque (présence de numérique, participation accrue des publics, prédominance de l'image...). En multipliant les accroches visuelles et en élaborant des supports riches en interprétations, au travers de références explicites et implicites à des imaginaires communs, l'institution a augmenté considérablement ses chances de marquer les esprits, bien qu'elle n'ait pas vraiment mobilisé l'argument événementiel de ses 150 ans, pourtant une force. Cette fictionnalisation a servi de fer de lance à la presse, notamment auprès des partenaires, qui ont repris l'univers de l'exposition pour habiller leurs articles, tout en l'enrichissant.

Cette stratégie communicationnelle comprenait toutefois un risque. En effet, il pouvait exister une confusion entre les connaissances "officielles" transmises au sein de l'exposition et les informations "officieuses" portées par l'ambiance fictionnelle et les références à des imaginaires partagés, marquées par le sceau de l'émotion. Nous avons pu 
relever une volonté de mettre le corps et l'expérience sensorielle au premier plan. Cette sollicitation a entraîné une forte participation du visiteur, qui construisait ainsi activement son expérience de visite et son acquisition de connaissances. Dans cette optique, le visiteur n'était plus seulement spectateur, mais se rapprochait de ce que Pierre Barboza nomme "spect@cteur" dans son propos sur l'hyperfiction ${ }^{5}$. De même, la composition dynamique des documents de communication, conçue pour des "lectures en Z" (suivant un modèle canonique des publicités commerciales), où les nombreuses zones de textes créent du mouvement, accroît la sensation d'aventure en laissant choisir au lecteur/spectateur le parcours à suivre. On pouvait néanmoins se demander si ces choix communicationnels n'étaient pas en contradiction avec l'image de l'institution muséale qui incarne une structure dépositaire des savoirs "légitimes", suivant des logiques stables et pérennes. Laquelle doit de surcroît répondre à ses fonctions de conservation et de transmission. En outre, l'ordonnancement associé à pareil établissement peut être contradictoire avec l'utilisation que le muséum fait du web et des réseaux sociaux, synonymes d'instantanéité et de flux. Une approche qui a manqué de clarté et aurait pu participer davantage à la fictionnalisation de l'exposition, mais aussi et potentiellement à sa promotion. Comme le souligne les résultats de l'enquête nationale sur la satisfaction des publics des musées nationaux, À l'écoute des visiteurs 2012 : "parler de sa visite en utilisant les réseaux sociaux en ligne devient une pratique courante, cette action est envisagée par $32 \%$ des visiteurs", une proportion en hausse depuis l'enquête citée réalisée en 2010 ( $25 \%$ des visiteurs avaient l'intention de parler de leur visite sur un média en ligne).

Pour créer un aspect ludique et participatif qui s'inscrit dans une dynamique moderne, comme ici cette odyssée instructive et transgénérationnelle visant à sortir le spectateur de sa dimension passive, il ressort finalement qu'un musée doit effectuer un fin jonglage entre visées informatives et visées ludiques, tant dans sa communication que dans ses choix scénographiques.

\section{Horizons d'attente}

D'après les résultats obtenus auprès de 10000 visiteurs interrogés dans 58 établissements, dont 47 musées nationaux, l'enquête nationale sur la satisfaction des publics (des musées nationaux) À l'écoute des visiteurs 2012 précédemment citée établit "trois indicateurs dont la complémentarité ainsi que la concordance de certaines modalités paraissent de nature à formaliser le niveau de satisfaction générale des visiteurs". En tête sur le podium : la relation entre l'expérience de visite et l'horizon d'attentes, dont la conformité des deux satisfait $64 \%$ des visiteurs. 


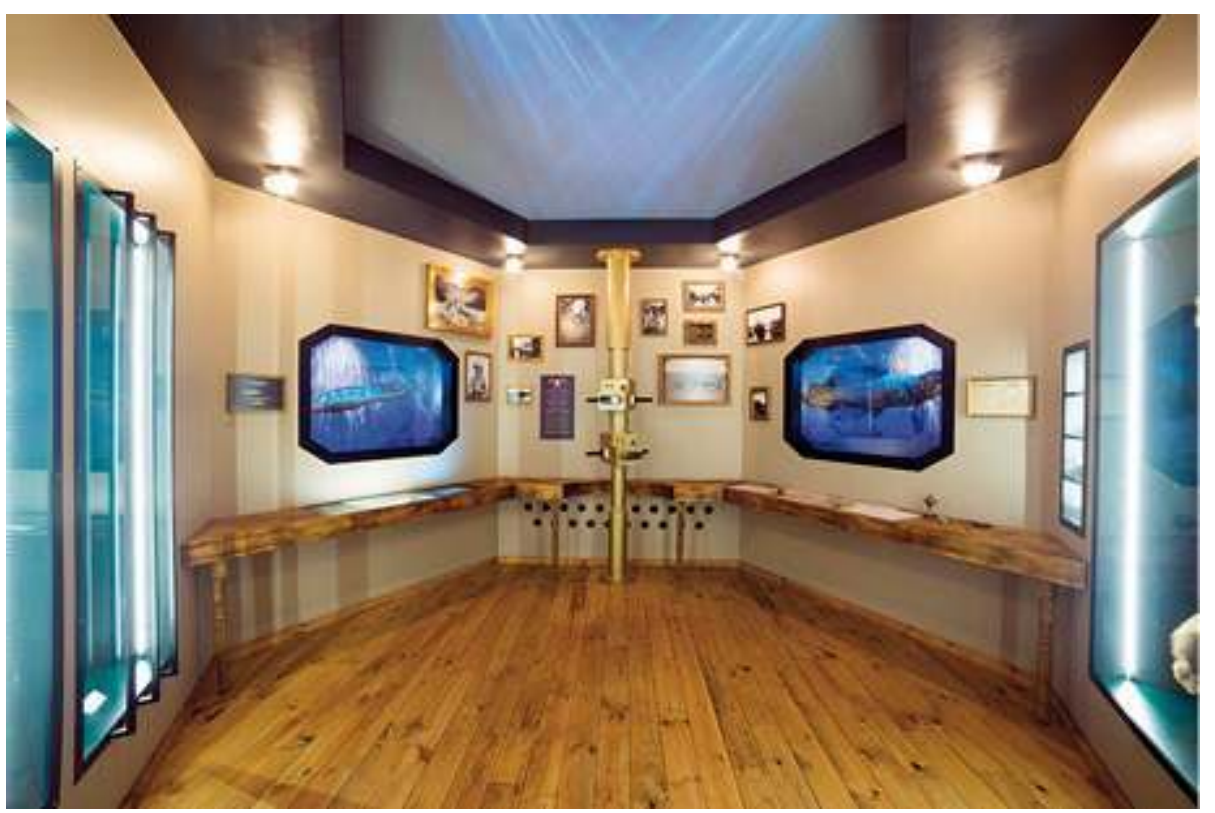

(c) Muséum de Toulouse/Jacques Sierpinski

L'espace "La crypte"

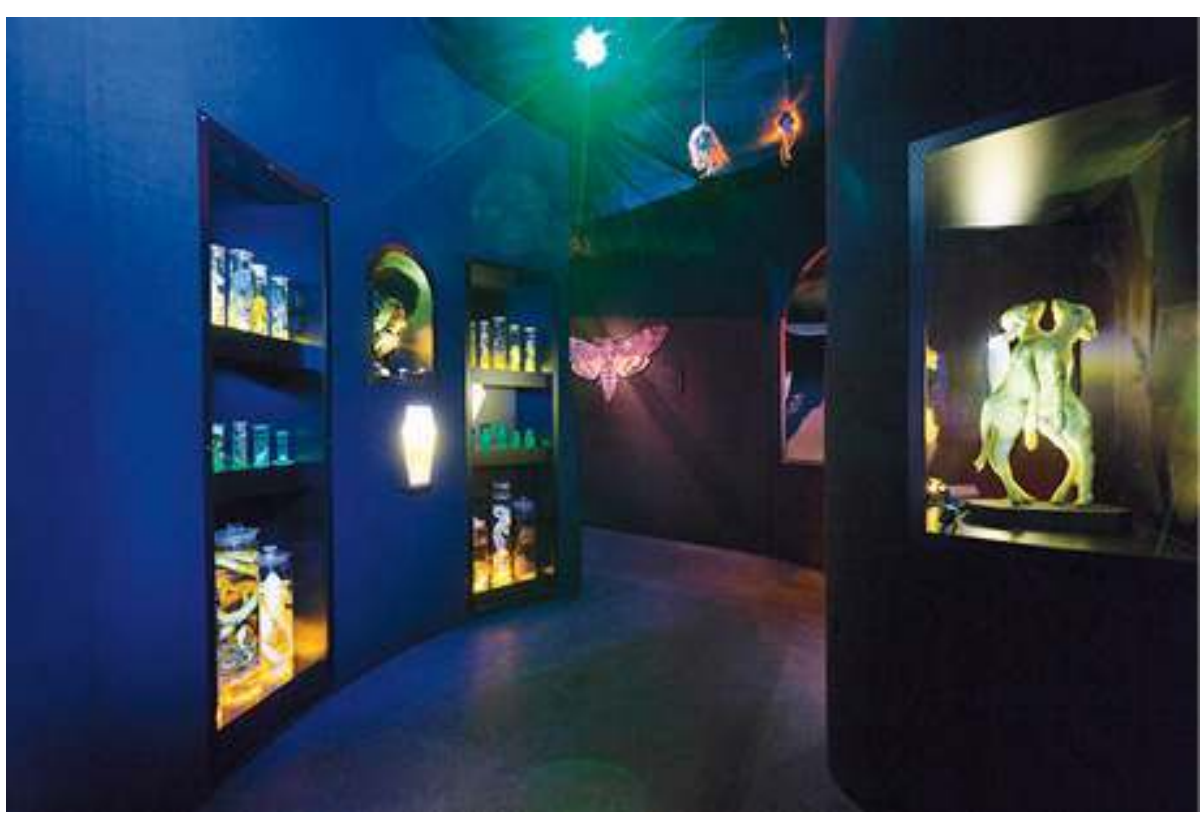

(c) Muséum de Toulouse/Jacques Sierpinski

11 Pour pouvoir analyser ici les horizons d'attente, expériences de visite et écart de représentation, nous avons défini plusieurs critères de sélection des publics étudiés. Nous nous sommes intéressés aux familles composées d'au moins un enfant de 8 ans et habitant en région Toulousaine. Nous réalisions un premier questionnaire dans la file d'attente de la billetterie du muséum, en isolant quelque peu les interrogés pour éviter que les réponses des uns influencent celles des autres. Et tous les membres de la famille étaient interrogés. Avec deux familles, nous avons réalisé l'entretien post-visite "à chaud", dans 
le café du muséum. Pour deux autres, l'entretien s'est déroulé une semaine plus tard. Deux bilans de visite se sont alors présentés à nous : les premiers entretiens étaient marqués d'exemples et détails précis concernant la visite, cependant que les seconds, moins axés sur le contenu de cette dernière, revêtaient une dimension plus réflexive, conséquence du temps écoulé entre ladite visite et notre deuxième rencontre : un délai propice à une prise de recul renforcée par des échanges entre les visiteurs.

Il ressort en premier lieu que les horizons d'attentes des visiteurs ne sont pas systématiques. Comme un clin d'œil aux travaux d'Emmanuel Ethis portant sur les publics des salles multiplexes ${ }^{6}$, certains visiteurs n'ont pas d'attentes prédéfinies lorsqu'ils vont au musée : ils n'ont souvent pas réfléchi à ce qu'ils voulaient/s'attendaient à voir et ne se sont, pour la plupart, même pas posé la question. Par conséquent, ayant vécu l'expérience sans point de comparaison, à l'issue de leur visite, ils ne peuvent qu'être satisfaits de ce qu'ils y ont vu ou découvert. Chez plusieurs autres personnes interrogées, les horizons d'attente furent créés de toutes pièces lors de l'entretien effectué au préalable. En effet, lorsque nous avons demandé explicitement aux visiteurs leurs attentes par rapport à leur future visite, certains les ont inventés. Ils nous décrivaient alors une expérience vécue qu'ils projetaient sur celle à venir pour lui donner une forme connue, cependant qu'elle n'aurait pas d'influence sur la réception de la visite. Concentrés sur leur ressenti présent, ils se sont finalement laissés surprendre par ce qu'ils ont vu, et sont sortis satisfaits. À l'inverse, certains visiteurs qui avaient également en mémoire d'autres expériences de visite vécues n'ont pas pu s'empêcher de les comparer avec l'exposition Les Savanturiers. Ils ont donc été particulièrement déçus par leur visite car il existait un véritable écart entre l'horizon d'attentes qu'ils s'étaient dessiné, fondé sur leurs connaissances et leurs expériences antérieures des musées, et leur expérience de visite effective. Certaines familles ne nous avaient pas fait part d'attentes particulières lors du premier entretien, or leur horizon était malgré tout bien défini. Interrogées une ou deux semaines après leurs visites, le temps de réflexion et la mise en commun de leurs impressions ont induit une homogénéisation de leurs points de vue, rappelant la tendance d'adaptation à l'impression dominante générale.

Les attentes apparaissaient parfois aussi au cours même des visites, car les visiteurs ont malgré tout été conditionnés par leurs expériences et leurs connaissances personnelles, leur vie privée, mais également par leurs visions préalables du musée, soit des facteurs purement subjectifs. Les visiteurs avaient ainsi des attentes dont ils ne prenaient pas forcément conscience avant d'être confrontés à l'exposition en elle-même. 


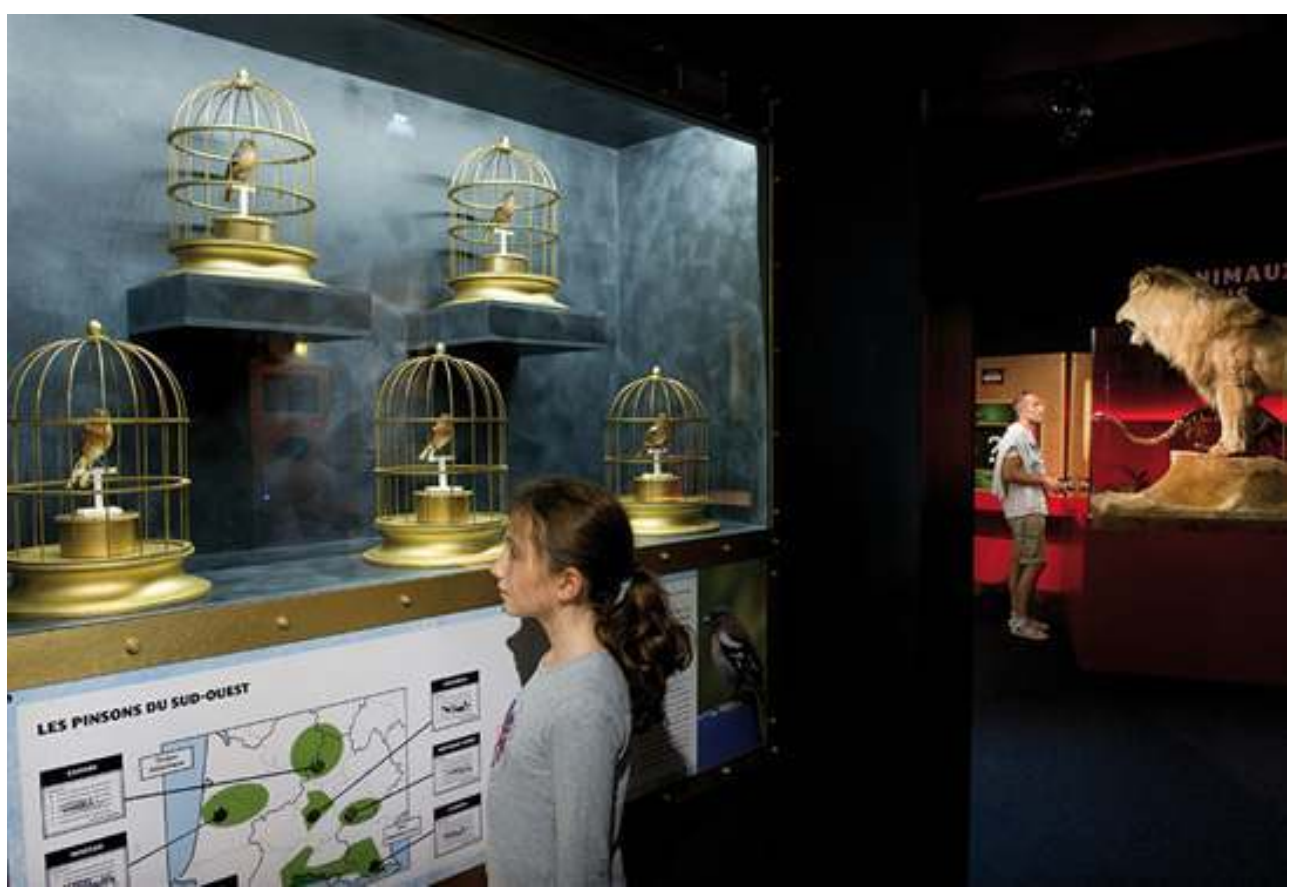

(c) Muséum de Toulouse

14 Enfin, nous avons observé un quatrième cas de figure parmi notre échantillon, qui s'appliquait notamment aux enfants interrogés. D'après leurs témoignages, ils ont été complètement satisfaits de l'exposition et ont trouvé qu'elle correspondait à leurs attentes. Cette satisfaction est cohérente par rapport à ce qu'ils avaient exprimé lors de l'entretien préalable : tous voulaient voir des animaux, faire des expériences, souhaitaient des paysages reconstitués et un décor attrayant et ludique. Autrement dit des attentes très visuelles et "faciles" à combler. Cela relevait alors davantage d'une certaine curiosité à découvrir des choses nouvelles, que d'attentes plus significatives. Ils ont donc été satisfaits de retrouver ces éléments lors de leur visite et d'être immergés dans des ambiances très différentes en fonction des salles. Leur horizon d'attente concernant leur vision des "savants-aventuriers" s'est également bien calqué sur leur visite effective. Cet engouement général de la part des enfants plus que des adultes, peut s'expliquer par le fait que leurs attentes sont moins exigeantes en terme de contenus, et moins imprégnées de culture et d'expériences antérieures. De plus, les enfants, par leur jeune âge, ont davantage de difficultés à remettre en question l'institution muséale : ils sont donc plus à même de se laisser surprendre et émerveiller par l'exposition et ses décors hauts en couleur.

\section{Parcours de visite}

Après avoir recensé les différentes salles et les différents dispositifs au cours d'une visite libre, nous avons observé deux familles de manière anonyme pour appréhender au mieux notre réflexion à propos des logiques de visite. Dans un second temps, nous avons équipé quatre familles de "lunettes caméra", en proposant une paire à un des adultes, et à un des enfants. Avec cet outil, nous avons pu analyser précisément les parcours de visite, 
l'utilisation des dispositifs, mais aussi les interactions entre visiteurs de manière intergénérationnelle. Cette dernière démarche s'est déroulée durant quatre dimanches après-midi du mois de janvier 2016.

Pour cette exposition, le muséum avait fait le choix de ne pas imposer de parcours. Ce choix nous a d'abord questionnés sur la façon dont le public allait l'appréhender et construire son expérience de visite. En effet, le visiteur pourrait rencontrer des difficultés, se sentir perdu, ou bien se retrouver à occulter inconsciemment certaines salles ou certaines parties d'entre elles, faisant ainsi une visite très peu exhaustive. Cette première hypothèse s'est couplée avec une seconde qui interrogeait les dispositifs numériques et manuels proposés au sein de l'exposition. Ceux-ci auraient plus ou moins de succès selon leur nature : les dispositifs numériques seraient davantage sollicités et appréciés que les plus littéraires. Mais qu'en était-il réellement de cette appréhension du parcours de visite et des dispositifs par les publics?

Au bas des escaliers menant à l'exposition, un dépliant indiquait le plan de celle-ci et le contenu des différentes salles. Nous nous sommes vite rendus compte que ce dernier semblait être plus une source de confusion pour les publics - les flèches pouvant indiquer un sens de visite tournaient sur elles-mêmes - qu'un support indispensable. Les différentes familles observées ne se sont pas saisies de ce support durant leurs déambulations, par choix ou par inadvertance. L'absence explicite de sens de visite ne leur a donc pas paru gênante. De plus, l'occultation de certains espaces ou de certaines salles fut perçue différemment pour chaque famille. Et la présence de médiateurs dans l'exposition a eu un rôle efficace pour les plus dubitatives à la recherche d'indications explicites sur le parcours de visite, ainsi que pour répondre à des demandes ponctuelles.

Exemple de dispositif interactif présent dans l'exposition

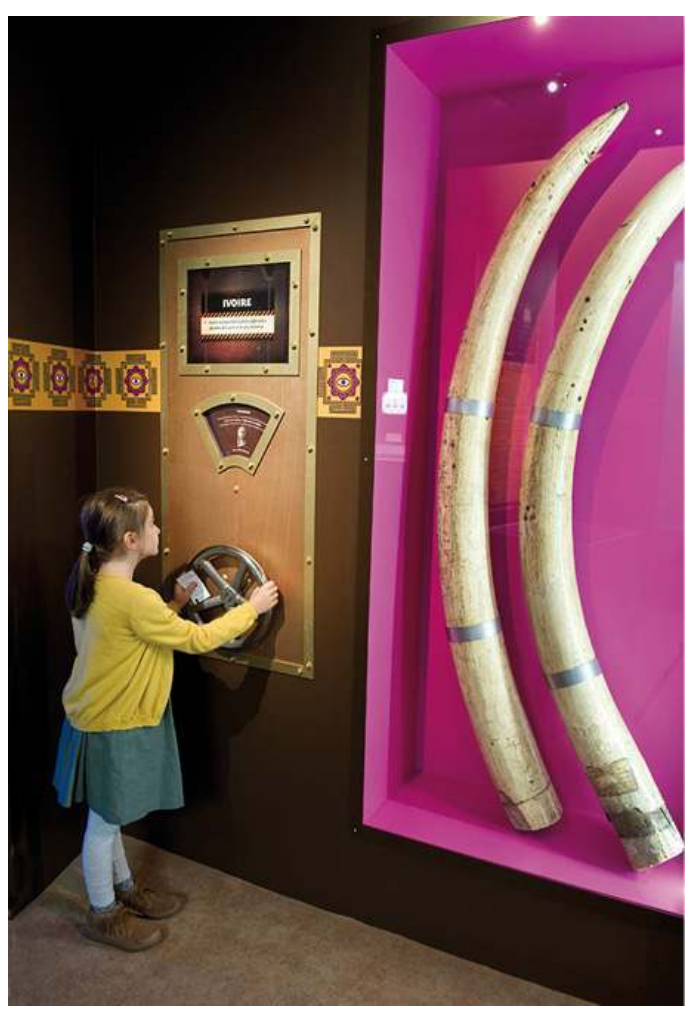

(c) Muséum de Toulouse 
Pour étudier les dispositifs, numériques et manuels, ainsi que leurs utilisations au sein de l'exposition, nous les avons répertoriés et classés selon le système suivant : interactifs pour ceux qui demandaient une action dite physique de la part du visiteur pour les faire fonctionner, et spectatoriels pour ceux qui n'en demandaient pas. Il a été constaté que les dispositifs numériques les moins sollicités présentent un inconvénient commun : ils étaient positionnés à une hauteur intermédiaire, ne correspondant ni aux adultes qui devaient trop se baisser pour voir le documentaire, ni aux enfants dont le champ de vision n'atteignait pas l'écran dans la plupart des cas.

19 De plus, tous avaient l'air moins captivés par un documentaire à écouter patiemment (dispositif spectatoriel), que par un écran nécessitant une interaction tactile et ludique (dispositif interactif). L'approche dite de design for all ou conception universelle est ici occultée, alors que s'il est bien conçu, un dispositif de médiation numérique peut favoriser l'accès aux œuvres pour une pluralité de publics ${ }^{7}$. L'information devait sembler trop longue à être délivrée lors d'un film documentaire, tandis que les dispositifs numériques, tels que les jeux, ont suscité un fort intérêt chez les petits comme chez les grands. "Le Vaisseau", qui met en scène la reconstitution d'un tigre en 3D, a été très apprécié par les plus jeunes, bien qu'il fût un dispositif purement spectatoriel et que les boutons destinés à contrôler l'animal étaient factices.

20 En ayant travaillé sur ce terrain constitué de familles, un troisième cas d'étude s'est présenté à nous au cours de nos observations : les parents endossant le rôle de médiateurs. En effet, nous avons pu noter que si le médiateur présent était peu sollicité par le visiteur adulte, c'est parce que l'adulte lui-même tenait ce rôle auprès de son enfant. Tous les adultes observés lors des visites faites avec les "lunettes caméra" nous ont semblé avoir un comportement similaire : ils posaient des questions afin d'intéresser l'enfant... lorsque ce n'était pas l'enfant lui-même qui demandait des explications. Ce rôle pédagogique semblait souvent venir "naturellement" au visiteur adulte accompagné de son enfant, si bien que ce pourrait être un critère à prendre en compte et à développer par le muséum pour améliorer cette médiation familiale.

\section{Médiations et médiateurs(trices)}

21 Dans cette partie de l'étude, nous nous intéressons aux rapports humains publicsmédiateurs, et particulièrement aux ressentis des médiateurs vis-à-vis de l'exposition. Pour articuler notre réflexion, nous avons réalisé une série d'entretiens semi-directifs individuels avec 11 des médiateurs du muséum, puis, à la suite de leur entretien, nous les avons observé en situation de médiation afin de confronter leur discours. Cette étude s'est déroulée sur 5 jours durant les mois de janvier et février 2016, à raison de 3 jours en semaine et 2 en week-end.

22 Le rôle du médiateur au sein de l'institution, ainsi que son rapport avec l'exposition Les Savanturiers est essentiel car il représente le muséum et transmet les savoirs du lieu. De ce fait, nous avons questionné l'implication et la prise en compte des médiateurs dans la conception de l'exposition, mais aussi le discours relatif à cette dernière, et la manière dont les médiateurs l'ont construit, se le sont approprié et l'adaptaient en fonction des différents publics. Enfin, nous nous sommes intéressés au maraudage et notamment la manière dont celui-ci était géré par les médiateurs ${ }^{8}$. Durant la conception de l'exposition, les médiateurs sont consultés par le biais de référents mais ne participent pas 
directement aux prises de décisions, ce que la plupart regrettaient car ils sont les professionnels du muséum in situ à avoir le plus de contact avec le public. L'efficacité du travail préparatoire d'appropriation de l'exposition était alors remise en question, malgré des démarches pour les impliquer et les renseigner, telles que des visites avant l'ouverture au public. Or, ces instants n'ayant pas été banalisés, des médiateurs n'ont pas pu y assister du fait de leur emploi du temps. Lors de nos entretiens, certains n'avaient pas encore pu la découvrir. Un décalage s'était alors créé avec les autres médiateurs. En outre, la richesse et la complexité de cette exposition ajoutaient une difficulté supplémentaire à la compréhension du contenu et des informations, donc à la transmission auprès des publics. La forte cohésion et les nombreux échanges entre les médiateurs permettaient cependant de compléter les informations fournies, selon les savoirs de chacun, pour que toutes et tous se saisissent au mieux de l'exposition. Cette stratégie de partage et de co-construction entre médiateurs a permis de créer une certaine horizontalité des échanges. Le médiateur a pour rôle principal de construire un discours à transmettre aux publics, lequel est nourri par les informations brutes que lui ont données les concepteurs de l'exposition. Les pré-connaissances de certains, mais surtout l'enrichissement à partir d'échanges collectifs, permettent d'améliorer leurs communications. La compréhension étant leur première préoccupation, les médiateurs travaillent d'autant plus à l'adaptation des discours en fonction des publics (le nombre de personnes, leurs âges, le parcours...). Nous avons constaté que le choix du vocabulaire utilisé constituait l'adaptation la plus courante, notamment avec le jeune public, et plus encore pour cette exposition qui n'était pas principalement destinée aux enfants. La fréquentation étant essentiellement familiale, les médiateurs se devaient de faire interagir les enfants pour intéresser les parents. En privilégiant l'échange avec l'un des deux, ils prenaient toutefois le risque de perdre l'attention du second. Pour résoudre ce problème, le dispositif du "caddie" permettait d'introduire un sujet, et de parler de l'exposition en présentant les objets qui le composaient. Ainsi, les enfants étaient captivés et le médiateur pouvait mobiliser du temps de parole pour les personnes souhaitant approfondir le propos. Cependant, il existait une réelle difficulté quant au fait de passer d'un discours à l'autre : les médiateurs n'étant ni des spécialistes de la petite enfance, ni des scientifiques, mais endossant les deux rôles. 
Le lion des cavernes qui revient à la vie en 3D dans l'espace "Le vaisseau".

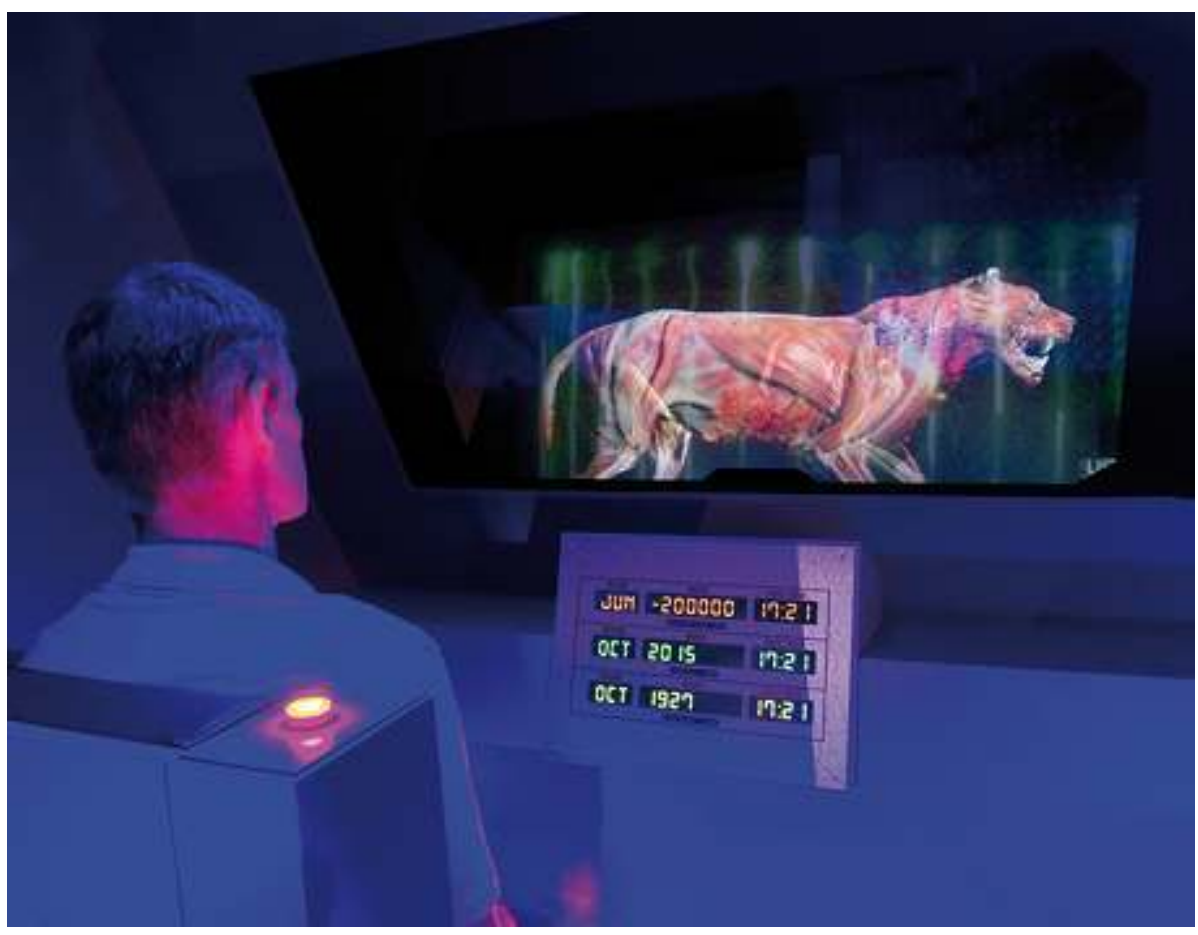

(c) Muséum de Toulouse

23 Les médiateurs du muséum devaient réaliser à tour de rôle des heures de maraudage dans l'exposition temporaire. D'après les entretiens, leurs techniques pour être visibles auprès des publics et ainsi favoriser un premier contact étaient similaires. Selon l'aisance de chacun, soit ils se plaçaient en bas des escaliers pour aborder les visiteurs dès leur arrivée, soit ils déambulaient dans l'exposition afin de percevoir si certains visiteurs se questionnaient sur un élément de l'exposition. Pour autant, il ressort des observations un problème de visibilité des médiateurs en maraudage malgré le fait qu'ils portent tous un gilet affichant clairement le logo du muséum. D'une part, les visiteurs ne savaient pas qu'un médiateur était là et ne prenaient conscience de sa présence que lorsqu'ils le voyaient répondre à des questions. En ce sens, il est apparu que l'action de rentrer en contact avec le public attirait les autres visiteurs et créait une dynamique. Pour autant, dans d'autres cas, les publics semblaient envisager cette médiation comme une intrusion dans leur visite et semblaient gênés par cette démarche inhabituelle à leurs yeux.

Pour passer outre ces deux problématiques, le médiateur doit rester actif. Or c'est une tâche qui demande de la confiance en soi et une grande motivation, que seule la pratique sur le long terme permet d'appréhender. Pour pallier la timidité ou la peur d'être intrusif, le caddie apparaît comme la solution adéquate : il suscite la curiosité des visiteurs et le médiateur maîtrise le sujet sur lequel il souhaite faire reposer son discours. Malgré ces hypothèses et les dires relevés en entretien, nous avons observé deux comportements de médiateur véritablement opposés. L'un extrêmement actif, surtout auprès des enfants ; l'autre passif, voire fermé physiquement, qui installait une barrière avec le visiteur. Cette perception controversée du maraudage est donc intrinsèquement liée à l'état d'esprit et à l'implication du médiateur. Et le constat global témoignait d'une nécessité d'impliquer les médiateurs dans cette fonction essentielle en leur permettant d'avoir une visibilité, mais 
aussi de sensibiliser les publics, qui, à l'heure actuelle, ne semblent toujours pas acculturés à cette pratique.

\section{Conclusion}

Bien que l'étude repose sur un nombre restreint de sujets étudiés pour chacune des thématiques abordées, elle mobilise un retour réflexif des publics comme des acteurs du muséum, et permet de pointer certains aspects de la conception des expositions et de la médiation au sein de l'établissement. Par exemple en révélant des ressentis difficiles à verbaliser, notamment concernant la perception de l'activité de maraudage chez les médiateurs. On pourrait alors imaginer plusieurs initiatives pour prendre en compte ces ressentis, comme communiquer à l'entrée du muséum ou de l'exposition sur leur présence et leurs actions, pour les rendre plus visibles et réduire leurs appréhensions visà-vis de cette activité. Les impliquer davantage dans le processus de conception des expositions semble être aussi une réelle demande de leur part, et pourrait permettre une meilleure cohérence dans la réalisation de la communication autour de l'exposition, et donc dans la création des horizons d'attente. Enfin il serait pertinent de réfléchir à des pistes pour déléguer une partie du travail de médiation aux parents, compte tenu de leur souhait d'interagir avec leurs enfants et des difficultés qu'éprouvent les médiateurs à s'insérer dans les cellules familiales. Ce point a été pris en compte avant-même la présentation de notre étude auprès des équipes du muséum : un nouveau plan de visite avec un quizz destiné aux plus jeunes a vu le jour. C'est un exemple d'outil adéquat pour les parents-médiateurs. D'autres initiatives pourraient être développées dans ce sens et cette dernière témoigne d'une réelle politique d'auto-évaluation interne et permanente au muséum, montrant aussi les limites de la réalisation d'une étude figée à une période donnée.

Un père et sa fille devant l'imposant lion des cavernes dans l'espace "Les animaux disparus"

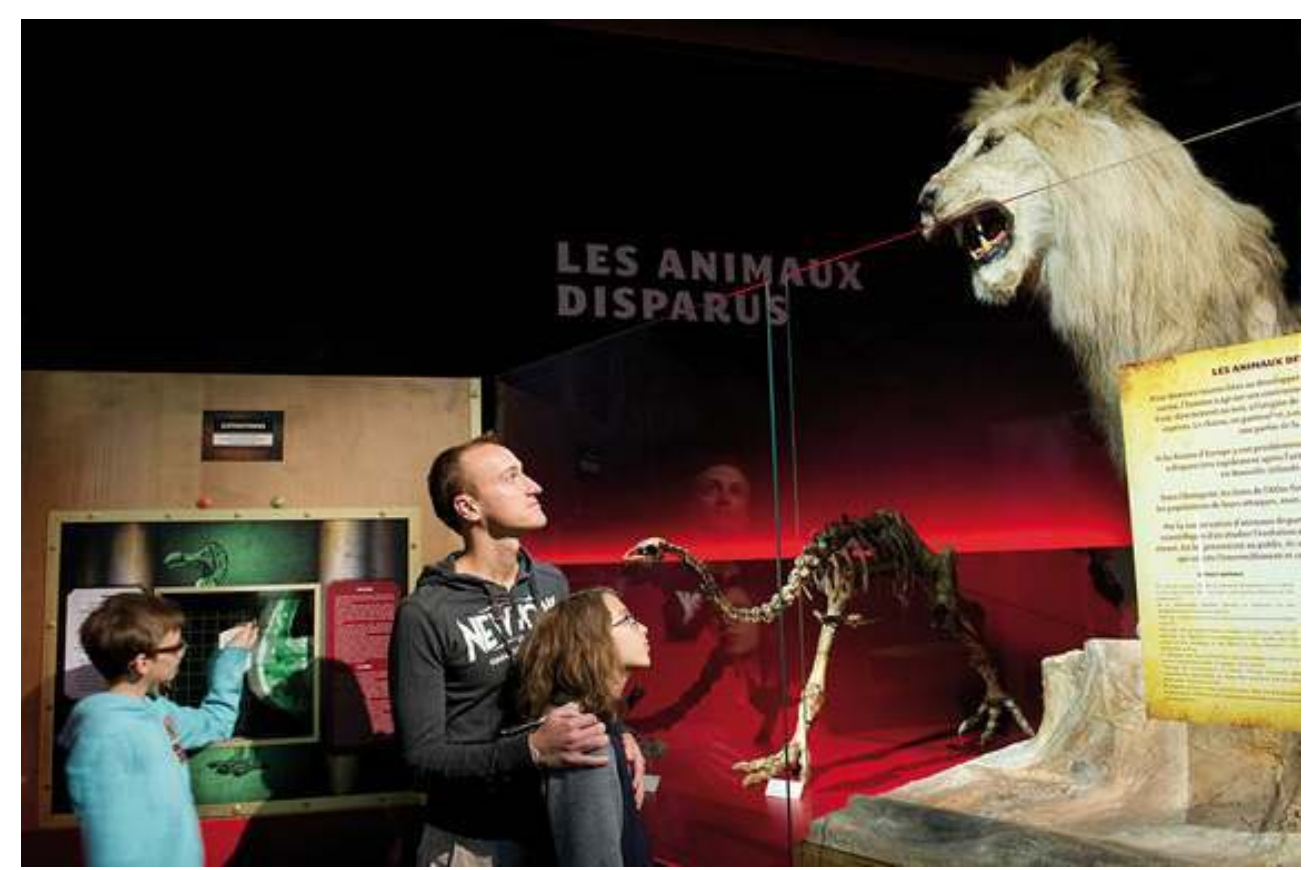

(c) Muséum de Toulouse 
26

Cette étude s'est révélée être une bonne occasion pour les étudiant(e)s d'inscrire des enseignements théoriques dans un cadre pratique, mais également un moyen pour le musée d'obtenir un regard extérieur, neuf, venant compléter qualitativement ses chiffres de fréquentation. Plus qu'un désir partagé de coproduction de connaissances, cette collaboration fut un enrichissement mutuel autour de la question des publics.

Cette analyse de l'exposition Les Savanturiers met en avant l'importance pour les musées et lieux culturels d'être en perpétuelle recherche d'innovations en matière de scénographie, de communication et de médiation. Elle montre également la nécessité pour ceux-ci d'impulser une vraie dynamique réflexive d'évaluation de leurs actions. Qu'elle soit réalisée par des professionnels ou des étudiants en voie de professionnalisation, chaque étude permet de faire émerger de nouvelles perceptions, de révéler de nouveaux faits et points de vue, ainsi que de partager des idées et des expériences. Mais tout l'intérêt d'une politique d'évaluation repose dans son approche qualitative, puisqu'elle est la seule à pouvoir appréhender certaines notions comme l'expérience de visite, les horizons d'attente ou bien faire naître des questionnements comme ici le rôle du parent-médiateur. Ainsi, on tend vers une réelle prise en compte des publics, des territoires et de toutes leurs caractéristiques associées : autrement dit vers un musée démocratique, à concevoir comme un partenariat avec divers publics et en accord avec la pensée selon laquelle "le musée tire souvent ses connaissances non pas des objets, ou des experts, mais des gens ordinaires"9.

\section{NOTES}

1. Davallon, J. Le musée est-il vraiment un média ? Publics et Musées, n², 1992. Regards sur l'évolution des musées (sous la direction de Jean Davallon), pp. 99-123.

2. Renoult, M. Le muséum de Toulouse reçoit le prix "Patrimoine et Innovation" pour l'expo Les Savanturiers, Actu Côté Toulouse.fr, 22 janvier 2016. http://actu.cotetoulouse.fr/museum-histoirenaturelle-toulouse-remporte-prix-patrimoine-innovation-exposition-savanturiers_28089.

3. "Depuis que la culture de l'évaluation a commencé de se diffuser dans le monde des musées au tournant des années 1980-1990, la question des indicateurs de la satisfaction des visiteurs s'est cristallisée avec la LOLF et la réforme statutaire qui a touché l'ensemble du secteur patrimonial dans la dernière décennie", in Eidelman, J. et Jonchery, A. À l'écoute des visiteurs résultat de l'enquête nationale sur la satisfaction des publics des musées nationaux. Ministère de la Culture et de la Communication, 2012.

4. L'étude présentée dans cette publication est le fruit d'une collaboration entre le département Art\&com de l'université Jean Jaurès à Toulouse et le muséum d'Histoire naturelle de Toulouse, née d'une volonté de renforcer le lien les unissant. Elle a été réalisée par Alric Amirault, Lucile Andri, Marine Chaumelle, Iris Cholvin, Benoît Descombe, Sarah Marchand, Laura Marnier, Lucie Plessard, Maud Taillade, Cheryline Ribes, Alice De Resseguier et Thomas Rokita, sous la direction de Muriel Lefebvre, Thibault Christophe et Jean-Marie Bodt.

5. Barboza, P. Fiction interactive "métarécit"et unités intégratives. L'image actée : scénarisations numériques. Paris : L’Harmattan, 2006, pp. 99-11.

6. Ethis, E. La caisse du cinéma : quand il faut décider, Communication et Langages, $\mathrm{n}^{\circ} 125,2000$. pp. 44-55.

La Lettre de I'OCIM, 173 | 2017 
7. Bougenies, F., Leleu-Merviel, S. et Sparrow, L. Effet captivant et apaisant de la médiation par tablette au musée : mesures physiologiques et motivationnelles, Études de communication, $\mathrm{n}^{\circ} 46$, 2016, pp. 87-108.

8. Éducation et action culturelle - Politique et activités (guide pratique). Service de soutien aux institutions muséales, ministère de la Culture, des Communications et de la Condition féminine, 2008.

9. Harris, J. dans Vers une redéfinition du musée ? sous la direction de Mairesse, F. et Desvallées, A. Paris : L'Harmattan, 2007, p. 63.

\section{RÉSUMÉS}

L'étude présentée ici montre que le traitement qualitatif de la réception d'exposition par les visiteurs et des rapports entre les publics et les médiateurs permet de développer une réflexion sur la médiation entre l'objet culturel et le visiteur mais également d'envisager une conception du musée comme un véritable partenariat avec les publics.

\section{INDEX}

Mots-clés : Exposition, médiation

\section{AUTEURS}

\section{ALRIC AMIRAULT}

étudiant en Master 2 Culture \& Communication

à l'université Toulouse 2 - Jean Jaurès

alric.amirault@gmail.com

\section{THOMAS ROKITA}

étudiant en Master 2 Culture \& Communication

à l'université Toulouse 2 - Jean Jaurès

thomas.rokita@gmail.com 